\title{
Study on vibration control system of structures based on magnetic levitation technology
}

\author{
Changan JIANG* and Satoshi UENO* \\ * Department of Mechanical Engineering, Ritsumeikan University \\ 1-1-1 Noji-higashi, Kusatsu, Shiga, 525-8577, Japan \\ E-mail: jiang@fc.ritsumei.ac.jp
}

Received: 12 June 2019; Revised: 30 August 2019; Accepted: 20 January 2020

\begin{abstract}
This study expresses a novel active vibration control method for structures by using magnetic levitation (MAGLEV) technologies. When a vibration occurs, a developed MAGLEV device will repeat to levitate and contact to the ceiling of structures properly. According to this movement, frictional force will be generated to consume vibration energy by the friction mechanism of the MAGLEV device. For making the MAGLEV device levitate stably, sliding model control method is employed. By varying levitation distance of the MAGLEV device, normal force which is related to friction can be adjusted. The experimental results show the effect of normal force on vibration control of structures, and verify the effectiveness of the proposed active vibration control method.
\end{abstract}

Keywords : Magnetic levitation(MAGLEV), Vibration control, Levitation, Friction mechanism, Sliding mode control

\section{Introduction}

In recent years, seismic base-isolation, vibration control and earthquake-resistant technology have been paid more and more attention with serious damage from the earthquakes, especially in seismically active countries such as Japan. In this research, seismic vibration control is focused on. Usually, seismic vibration control method can be broadly classified as passive, semi-active and active control approaches (Seto, 2006). The idea of passive vibration control method is to dissipate the vibration energy by using tuned mass damper (TMD) or energy-absorbing device (Sun et al., 1995) such as friction damper, fluid viscous damper etc. It is usually applied to protect the housing against earthquake (Housner et al., 1997; Haramoto et al., 2000; Spencer and Nagarajaiah, 2003). The advantages of this method are little maintenance and without external power supply. However, it will not provide satisfactory performance when characteristic of excitation varies. According to this problem, semi-active control method (Jalili, 2002; Nelson et al., 2015) was applied to adjust the stiffness or damping characteristics of semi-active device in real time with a low power input. As semi-active device is inherently passive, they cannot destabilize the system. In practice, semi-active control method is applied to skyscraper. In active control method, force actuator is employed for providing external energy to reduce vibration. It is usually used to reduce wind-induced vibration and transportation vibration. Active mass damper (AMD) is the most famous actuator for active vibration control. The related researches have been studied since 1980s, and the AMD system was successfully installed in the actual building 'Sendagaya INTES' in Tokyo, Japan (Yamamoto et al., 2001). The AMD is usually installed on the top of the building and driven to move its auxiliary mass for vibration control by additional actuators such as pneumatic actuator, hydraulic actuator etc. With development of magnetic levitation technology and control theory (Kamel et al., 2014; Gutierrez and Luijten, 2018), electromagnet based mechanism has been used for active vibration control instead of pneumatic actuator and hydraulic actuator because of its high-speed response (Dihoru et al., 2011).

Considering energy conservation, a new-style active vibration control device is developed by using magnetic levitation (MAGLEV), which is call MAGLEV device (Jiang and Ueno, 2016). It has hybrid magnets which are composed of permanent magnets and electromagnets. Normally, permanent magnets can make MAGLEV device adhere to iron plate on the ceiling of housing with zero electric power. When a vibration occurs, electromagnets can make MAGLEV device 
repeat to levitate and contact to the ceiling properly. By controlling the timing of switching between levitation state and contact state of MAGLEV device, frictional force will be generated to consume the energy of the vibration. For making the MAGLEV device levitate stably, sliding mode control method is employed in this research. By varying levitation distance of the MAGLEV device, normal force which is related to frictional force can be adjusted. With the variation of normal force, the effect of vibration control is also changed. In order to verify the effectiveness of the proposed active vibration control method and show the relationship between normal force and the effect of vibration control, simulation results are shown.

The rest of this paper is organized as follows. In Section 2, the composition of developed vibration control system and principle of vibration control are introduced. For consuming the energy of vibration, a MAGLEV device is developed to generate the frictional force at the contact point with the ceiling of the structure. In Section 3 , simulation results are shown to verify the effectiveness of the proposed method. In order to realize levitation control and guarantee that MAGLEV device can generate the desired normal force, sliding mode control method is employed and introduced in Section 4, and levitation test is done as a preliminary experiment to confirm whether the state of MAGLEV device can be switched between levitation and contact stably or not. The experimental results of vibration control are shown and analyzed in Section 5. Finally, conclusion of this paper is drawn in Section 6.

\section{Vibration control system of two-story structure}

\subsection{Two-story structure}

In this research, a two-story wooden structure model is considered as the plant which is shown in Fig. 1. On the ceiling of the 2nd floor, iron plate is attached for MAGLEV device docking. Parameters and material of each part of the structure model are shown in Table 1. The natural frequencies of 1 st and 2nd mode are measured as $2.01 \mathrm{~Hz}$ and 7.60 $\mathrm{Hz}$ according to the hammering test. Since a MAGLEV device is developed to generate frictional force for consuming vibration energy, it is considered into Fig. 2 which shows coordination for two-story structure model.

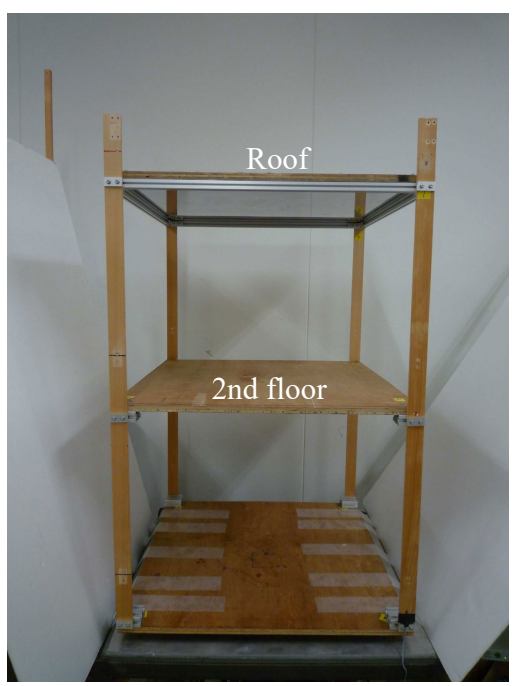

Fig. 1 Two-story structure model. The roof and floors are made of Plywood, pillars are made of cypress, the attached iron plate is made of SPCC.

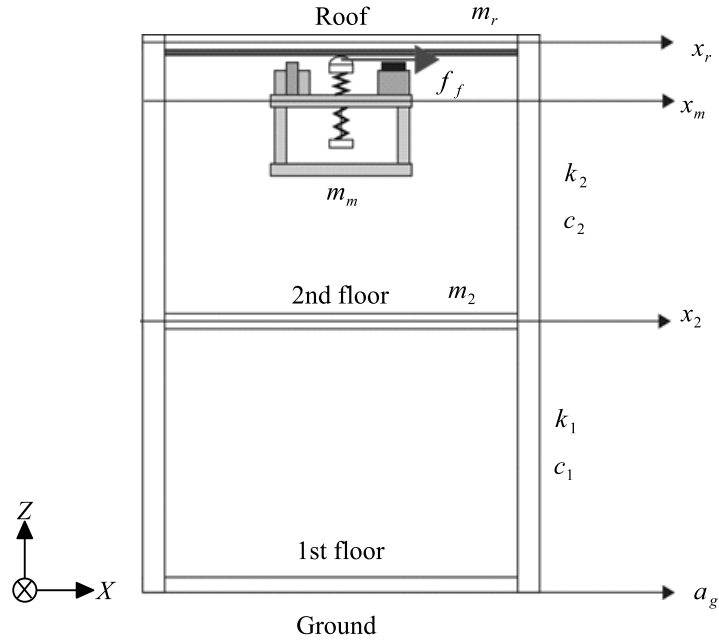

Fig. 2 Coordination for two-story structure model. Horizontal displacements of the structure and MAGLEV device are considered during vibration control.

Table 1 Parameters of the structure

\begin{tabular}{c|c|c|c|c}
\hline & Length [mm] & Width [mm] & Thickness [mm] & Material \\
\hline Roof & 550 & 550 & 12 & Plywood \\
Iron plate & 550 & 550 & 1 & SPCC \\
Floors & 550 & 550 & 12 & Plywood \\
Pillars & 800 & 30 & 5 & Cypress \\
\hline
\end{tabular}

Since only horizontal vibration is considered in this research, we use $x_{m}, x_{r}$ and $x_{2}$ to describe the displacements of MAGLEV device, roof and 2nd floor relative to the ground, respectively. $a_{g}$ denotes acceleration of the ground. $f_{f}$ denotes frictional force between MAGLEV device and the ceiling. According to this two-story structure model, the equation of 
motion is described as follows.

$$
M \ddot{X}+C \dot{X}+K X=E a_{g}+F f_{f}
$$

where

$$
\begin{aligned}
& X=\left[\begin{array}{l}
x_{r} \\
x_{2} \\
x_{m}
\end{array}\right] \quad M=\left[\begin{array}{ccc}
m_{r} & 0 & 0 \\
0 & m_{2} & 0 \\
0 & 0 & m_{m}
\end{array}\right] \quad C=\left[\begin{array}{ccc}
c_{2} & -c_{2} & 0 \\
-c_{2} & c_{1}+c_{2} & 0 \\
0 & 0 & 0
\end{array}\right] \\
& K=\left[\begin{array}{ccc}
k_{2} & -k_{2} & 0 \\
-k_{2} & k_{1}+k_{2} & 0 \\
0 & 0 & 0
\end{array}\right] \quad E=\left[\begin{array}{l}
-m_{r} \\
-m_{2} \\
-m_{m}
\end{array}\right] \quad F=\left[\begin{array}{c}
-1 \\
0 \\
1
\end{array}\right]
\end{aligned}
$$

$m_{m}, m_{r}$ and $m_{2}$ denote masses of MAGLEV device, roof and 2nd floor, respectively. $k_{1}, k_{2}$ and $c_{1}, c_{2}$ denote spring and damping coefficients of pillar of each floor.

\subsection{MAGLEV device}

For providing frictional force $f_{f}$ to consume the vibration energy, a MAGLEV device (see Fig. 3 ) is developed. The main parts of MAGLEV device are three hybrid magnets which are composed of permanent magnets and electromagnets (also see Fig. 3). Permanent magnets can make MAGLEV device adhere to the iron plate without any currents. Electromagnets are used to weaken the attraction of permanent magnets by modifying currents which flow in the electromagnets' coils. The friction mechanism is developed not only to provide a contact point to generate frictional force, but also to make normal force at the contact point be proportional to the levitation distance of MAGLEV device. Since two linear springs are employed in it, the normal force can be described as

$$
f_{n}=2 k_{s} \Delta z
$$

where $k_{s}$ is spring constant, $\Delta z$ is the deflection of MAGLEV device's friction mechanism.

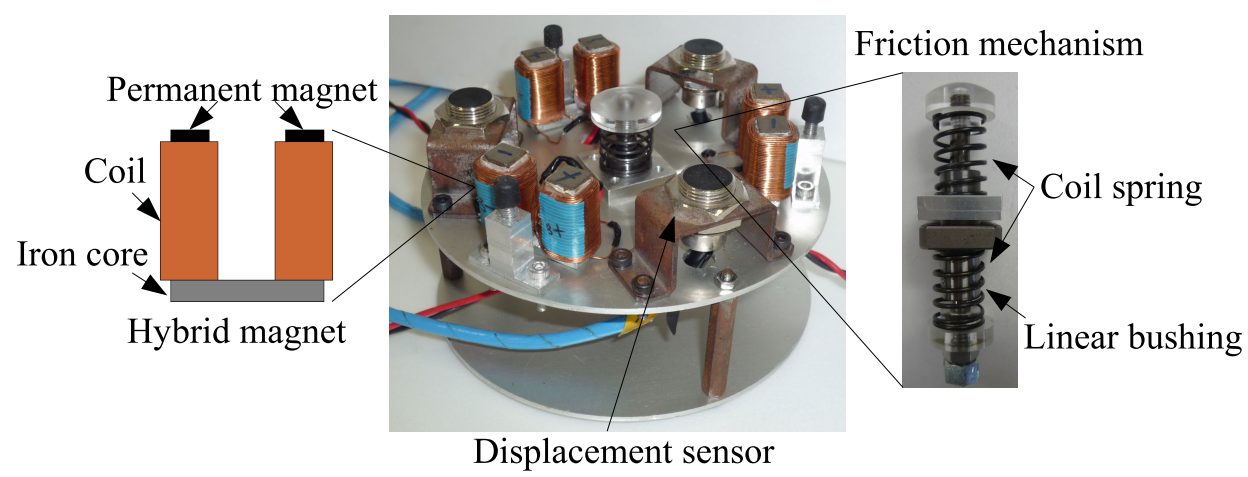

Fig. 3 MAGLEV device. It consists of one friction mechanism which is installed at the center, three each of hybrid magnets and eddy current displacement sensors which are installed at intervals of 120 degrees. One hybrid magnet is composed of an electromagnet and two permanent magnets which are fixed on top of the electromagnet. The friction mechanism has a contact point made of acrylic resin, a linear bushing and two coil springs whose spring constant $k_{s}=1 \mathrm{~N} / \mathrm{mm}$.

\subsection{Vibration control system}

The vibration control system for two-story structure model is designed as Fig. 4. Power amplifies (APEX PA01), sensor amplifies (SENTEC LS-500-4A) and strain amplifier (KYOWA DPM-711B) are employed to amplify the corresponding sensor signals, respectively. Levitation control and vibration control are implemented by using a DSP (dSPACE DS1104). According to the feedbacked sensor signals, the state of the MAGLEV device is switched between levitation and contact based on the proposed principle of vibration control. The levitation distance of the MAGLEV device is achieved by using the designed sliding mode levitation controller, which will be introduced in detail in Section 4. In the next section, the principle of vibration control will be described with schematic diagram. 


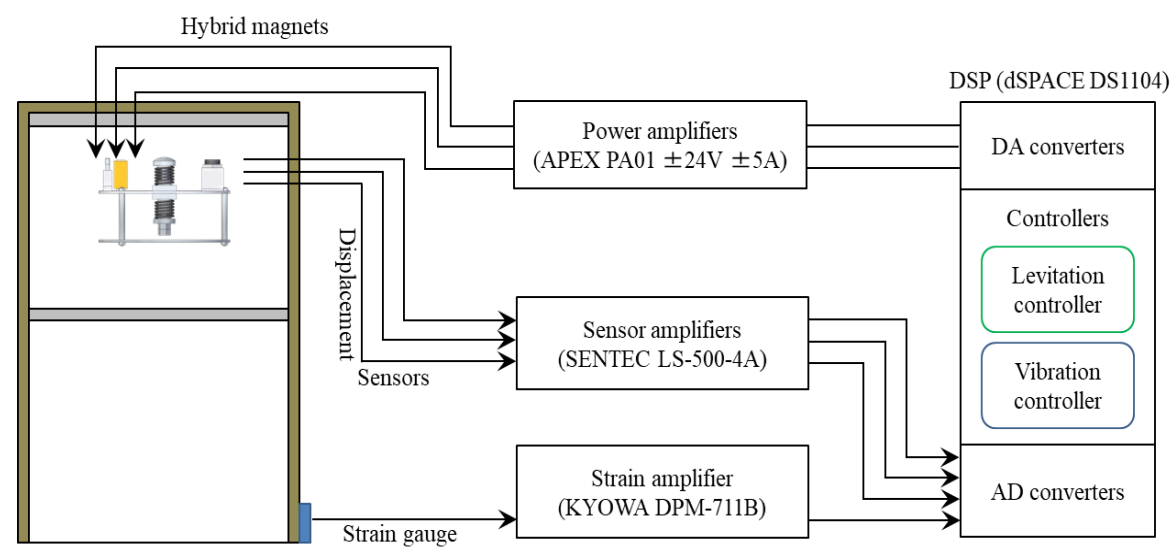

Fig. 4 Vibration control system. Levitation and vibration control was realized by using a digital signal processor (DSP). The feedback signals captured by displacement sensors and strain gauge were transmitted through the corresponding amplifiers to AD converters of DSP, respectively. The control currents were outputted from DA converters of DSP to the coils of hybrid magnets by passing through the power amplifies.

\subsection{Principle of vibration control}

Normally, permanent magnets which are installed on the MAGLEV device make it adhere to the iron plate on the ceiling of structure with zero electric power. When a vibration occurs, MAGLEV device will repeat to levitate and contact with the iron plate properly. In Fig. 5, principle of vibration control of a two-story structure model with the developed MAGLEV device is shown.

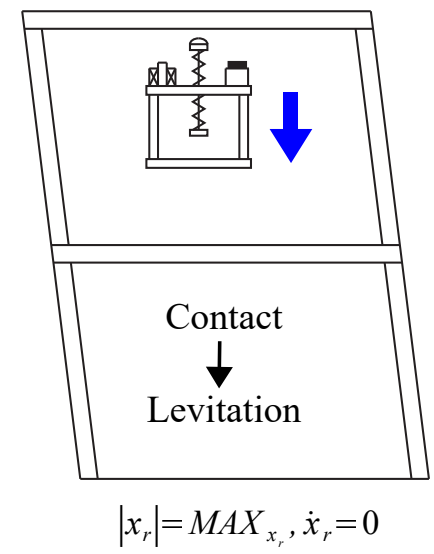

(a)

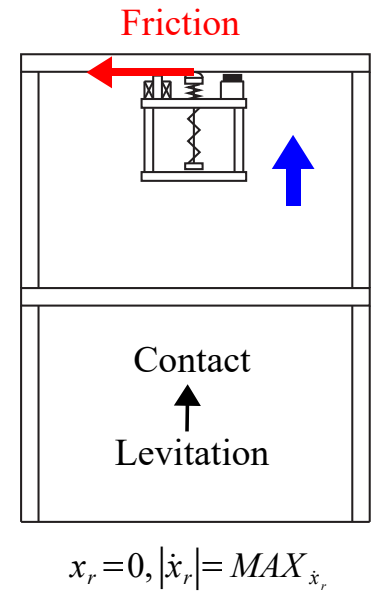

(b)

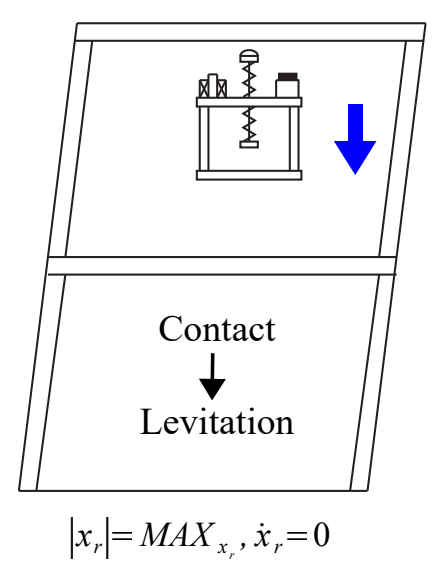

(c)

Fig. 5 Principle of vibration control. Blue arrows show the direction of the movement of MAGLEV device, red arrow shows the direction of the frictional force at the contact point. $x_{r}$ and $\dot{x}_{r}$ denote the displacement of the roof relative to the ground. $M A X_{x_{r}}$ and $M A X_{\dot{x}_{r}}$ denotes the maximum value of $x_{r}$ and $\dot{x}_{r}$, respectively.

In Fig. 5 (a) (c), three moments of switching states of MAGLEV device are shown. While vibration occurs, the roof takes MAGLEV device to move to the maximum amplitude $\left(\left|x_{r}\right|=M A X_{x_{r}}\right)$ on the left side (see Fig. 5(a)). At this moment, both of the velocities of the roof and MAGLEV device are zero $\left(\dot{x}_{r}=0\right)$. Then we switch the state of MAGLEV device from contact state (contact to the iron plate with the friction mechanism) to levitation state (noncontact to the iron plate with the friction mechanism) and let it levitate by controlling the currents of hybrid magnets till the roof returns to the original place $\left(x_{r}=0\right)$ and the velocity of the roof becomes maximum $\left(\left|\dot{x}_{r}\right|=M A X_{\dot{x}_{r}}\right)$ (see Fig. 5(b)). The state of MAGLEV device is switched from levitation state to contact state by weakening the currents of hybrid magnets and frictional force whose expression was given in previous section is generated at the contact point. The vibration energy is consumed by the frictional force till the condition of relative velocity is satisfied as $\left|\dot{x}_{r}-\dot{x}_{m}\right| \leq v_{h}$. Then the roof will take MAGLEV device to move to the maximum amplitude on the right side (see Fig. 5(c)). The state of MAGLEV device will be switched again from contact state to levitation state by enhancing the currents of hybrid magnets. With repeated the above process, the vibration of two-story structure model can be reduced. 


\section{Simulation}

In order to verify the effectiveness of the proposed vibration control method, simulation was done by using MATLAB/Simulink which is a graphical programming environment for modeling and analyzing dynamical systems. For solving Eq. (1), we employed Runge-Kutta method by using the parameters (see Table 2) in the simulation. We added a pseudo-impulse disturbance whose amplitude is $1 \mathrm{~m} / \mathrm{s}^{2}$ in $0 \sim 0.1 \mathrm{~s}$ to $a_{g}$ to make two-story structure model vibrate. During the vibration control, we set the normal force $f_{n}=2 \mathrm{~N}$.

Table 2 Parameters in the simulation

\begin{tabular}{c|c|c|c|c|c|c|c|c}
\hline \multicolumn{3}{c|}{ Mass [kg] } & \multicolumn{2}{c|}{ Spring coefficient [N/m] } & Damping coefficient [Ns/m] & Coefficient of friction & Threshold velocity [m/s] \\
\hline$m_{r}$ & $m_{2}$ & $m_{m}$ & $k_{1}$ & $k_{2}$ & $c_{1}$ & $c_{2}$ & $\mu$ & $v_{h}$ \\
5.15 & 2.30 & 0.53 & 4500 & 1100 & 4 & 7 & 0.245 & $0.5 \times 10^{-3}$ \\
\hline
\end{tabular}

\subsection{Friction model}

In this research, we regard friction as Coulomb friction. So when relative velocity $\dot{x}_{r}-\dot{x}_{m} \neq 0$, the frictional force between MAGLEV device and the ceiling is

$$
f_{f}=\operatorname{sgn}\left(\dot{x}_{r}-\dot{x}_{m}\right) \mu f_{n}
$$

where $\mu$ is the coefficient of dynamic friction, $f_{n}$ is the normal force at the contact point.

When $\dot{x}_{r}-\dot{x}_{m}=0$, MAGLEV device and roof can be regarded as one, and

$$
f_{f}=m_{m} \ddot{x}_{r}+m_{m} a_{g}
$$

According to Eq. (1) we can obtain

$$
f_{f}=\frac{m_{m}}{m_{m}+m_{r}}\left(-k_{2} x_{r}+k_{2} x_{2}-c_{2} \dot{x}_{r}+c_{2} \dot{x}_{2}\right)
$$

In this research, we introduce a threshold velocity $v_{h}$ and assume that when the relative velocity is small $\left(\left|\dot{x}_{r}-\dot{x}_{m}\right| \leq\right.$ $\left.v_{h}\right), f_{f}$ is approximated to a saturation function

$$
f_{f}=\left\{\begin{array}{cc}
f_{f 0} & \left|f_{f 0}\right|<f_{d} \\
\operatorname{sgn}\left(f_{f 0}\right) \mu f_{N} & \left|f_{f 0}\right| \geq f_{d}
\end{array}\right.
$$

where, $f_{f 0}=\frac{m_{m}}{m_{m}+m_{r}}\left(-k_{2} x_{r}+k_{2} x_{2}-c_{2} \dot{x}_{r}+c_{2} \dot{x}_{2}\right)$. Assuming a static friction coefficient is equal to $\mu$, the maximum static frictional force becomes $f_{d}=\mu f_{n}$. When $\left|\dot{x}_{r}-\dot{x}_{m}\right|>v_{h}$, frictional force is described by Eq. (3).

In the simulation, the above expression of frictional force is employed. From the expression of frictional force, we know that the magnitude of frictional force can be modified by the normal force $f_{n}$, and the direction of frictional force is determined by relative velocity $\dot{x}_{r}-\dot{x}_{m}$.

\subsection{Simulation results}

Simulation results are shown in Figs. 6-9. In Fig. 6, the displacements of roof which were obtained with and without control are shown. In order to confirm whether the timing of state switching is correct or not, state of MAGLEV device is shown with blue solid line, where positive denotes state of contact and negative denotes state of levitation in Fig. 6. The timing of state switching is agreement with the proposed principle of vibration control which is shown in Fig. 5. Comparing the results of displacements, we can see the proposed control method is effective for reducing the vibration of two-story structure model.

In Figs. 7-9, relative displacement of MAGLEV device, relative velocity of MAGLEV device and frictional force are shown together with state of MAGLEV device, respectively. From Fig. 7, we know that MAGLEV device stopped at around $8.5 \mathrm{~mm}$ far away from the original position. Since this distance is quite smaller than the allowable range of MAGLEV device's movement, it is not considered further in this research. In Fig. 8, we can find that state of MAGLEV device was switched from levitation to contact when the relative velocity reached the maximum. It is also agreement with the proposed principle of vibration control. 


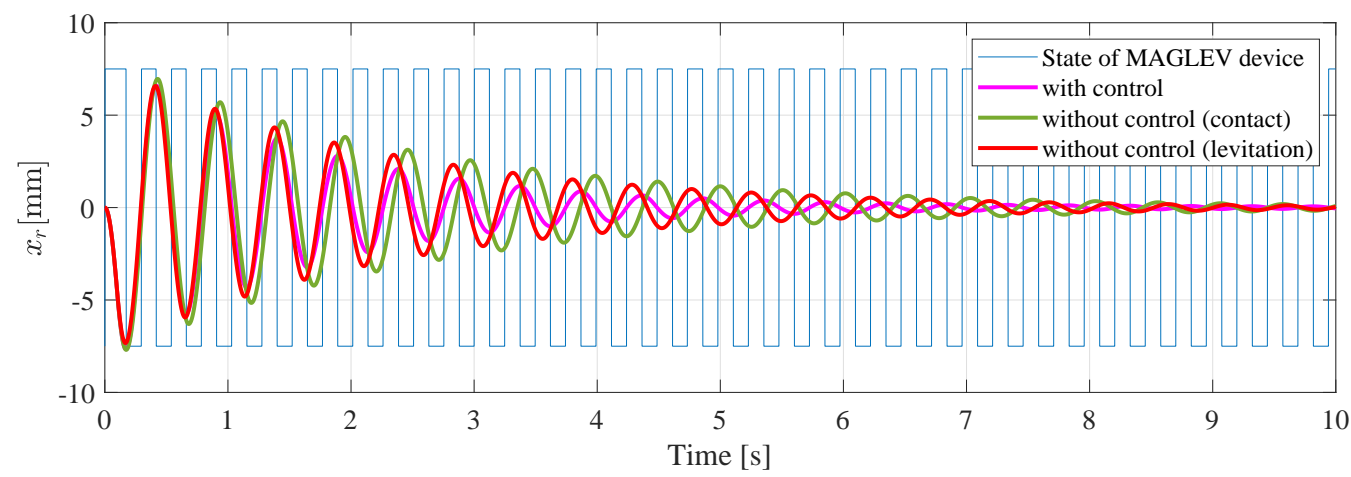

Fig. 6 Displacements of the roof. The comparison between the displacements with control and without control is shown. The state of MAGLEV device is also given to show the timing of state switching.

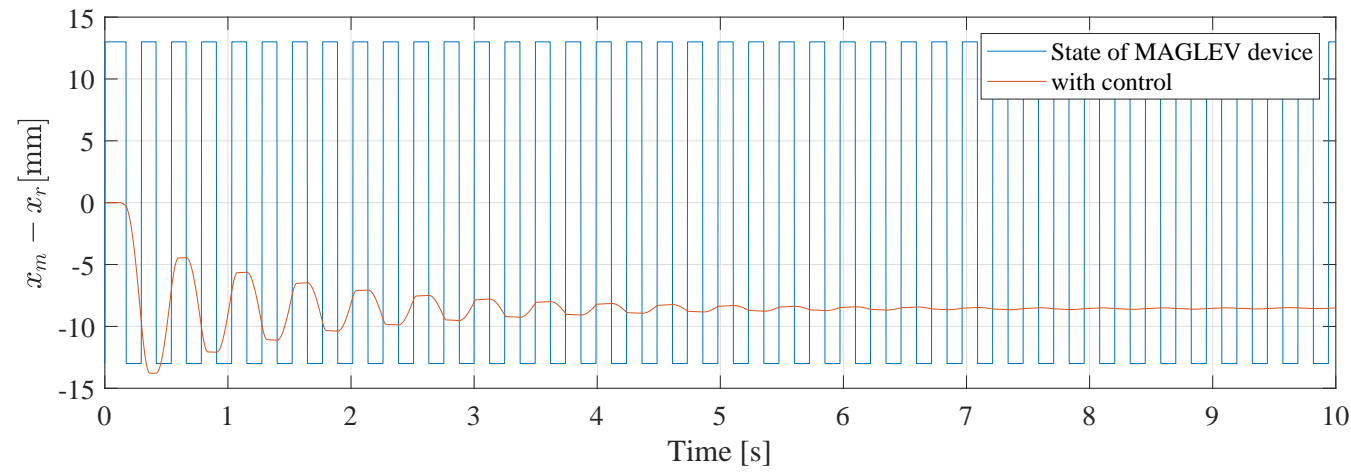

Fig. 7 Relative displacement of MAGLEV device. It stopped at around $8.5 \mathrm{~mm}$ far away from the original position.

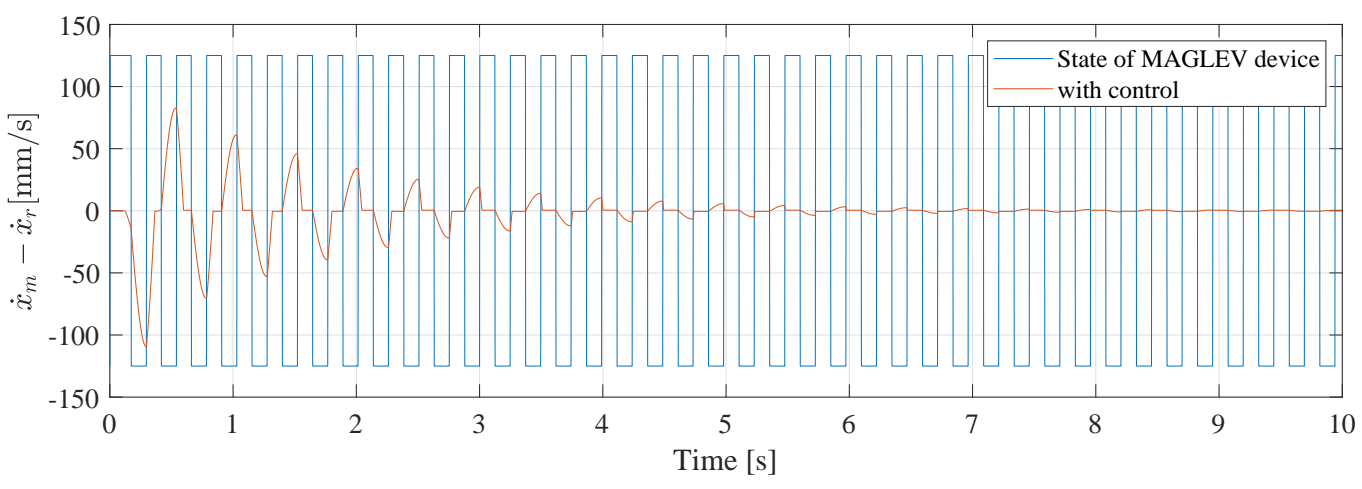

Fig. 8 Relative velocity of MAGLEV device. It increased when MAGLEV device was in levitation state, and decreased when MAGLEV device was in contact state.

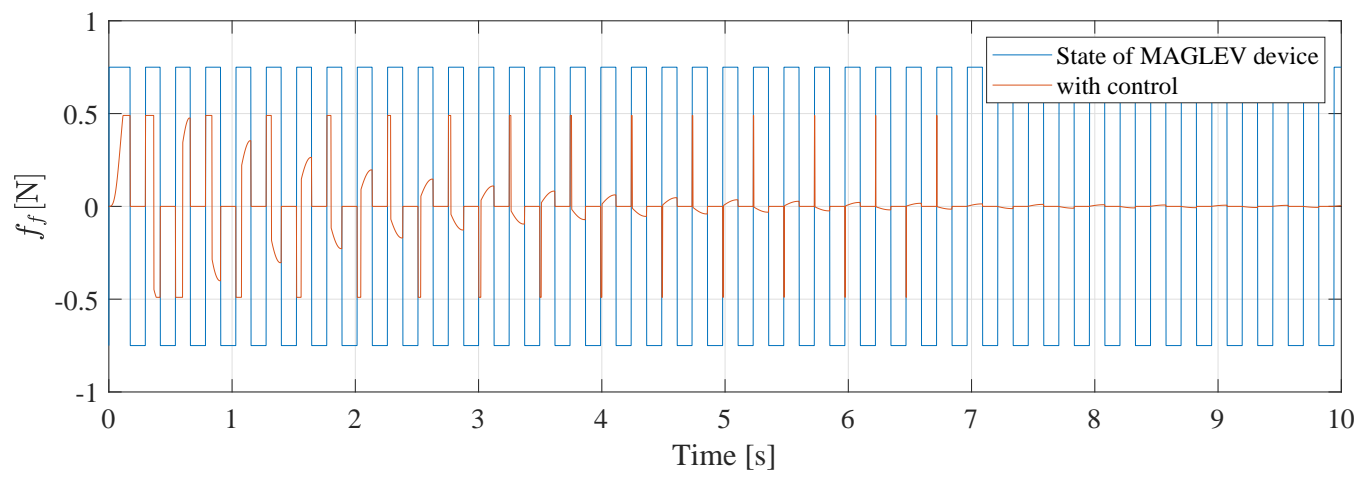

Fig. 9 Frictional force. Since normal force $f_{n}$ was set as $2 \mathrm{~N}$, the maximum frictional force was $0.49 \mathrm{~N}$ when MAGLEV device was in contact state. 


\section{Levitation control}

As mentioned in previous section, we know that the magnitude of frictional force can be decided by the normal force $f_{n}$. According to Eq. (2), we also know that the normal force can be modified by adjusting the deflection $\triangle z$ of MAGLEV device's friction mechanism. Hence, levitation controller is designed and introduced in the section.

\subsection{Sliding mode control}

In our considered system, the MAGLEV device levitates at various air gap lengths to modify the normal force at the contact point, then the characteristics of the MAGLEV device are variant. Moreover, the friction at the contact point acts as disturbance to the MAGLEV device. Therefore, a conventional PID controller could not stabilize the MAGLEV device very well. In order to keep the performance of levitation of the MAGLEV device during vibration control, sliding mode controller is designed for this system.

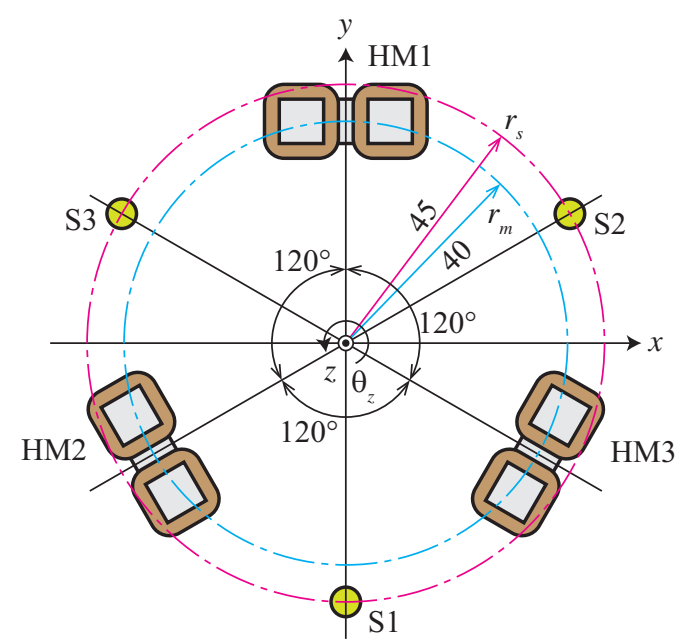

(a) Top View

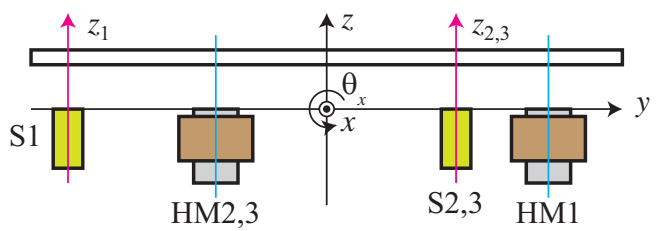

(b) $x$-Side View

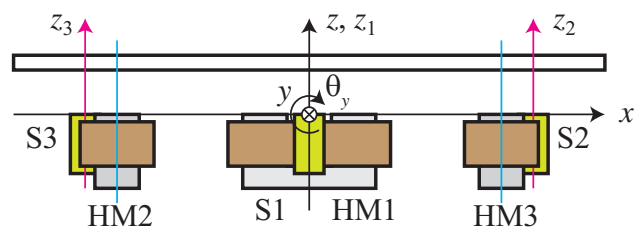

(c) $y$-Side View

Fig. 10 Coordinate system of the MAGLEV device. HM1 3 denote hybrid magnets, S1 3 denote displacement sensors, and $z_{1} \sim z_{3}$ denote the displacement of each sensor. $\theta_{x}$ and $\theta_{y}$ are the tile angles around $\mathrm{x}$ axis and $\mathrm{y}$ axis, respectively. $r_{m}$ and $r_{s}$ are the radii of the location of the hybrid magnets and displacement sensors.

The state and output equation of the MAGLEV device are described as follows according to the coordinate system of the MAGLEV device (see Fig. 10).

$$
\begin{aligned}
& \frac{d}{d t}\left[\begin{array}{l}
X_{L} \\
\dot{X}_{L}
\end{array}\right]=\left[\begin{array}{cc}
0_{3 \times 3} & I_{3 \times 3} \\
A_{L} & 0_{3 \times 3}
\end{array}\right]\left[\begin{array}{c}
X_{L} \\
\dot{X}_{L}
\end{array}\right]+\left[\begin{array}{c}
0_{3 \times 3} \\
B_{L}
\end{array}\right] U_{L} \\
& Y_{L}=\left[\begin{array}{ll}
C_{L} & 0_{3 \times 3}
\end{array}\right]\left[\begin{array}{c}
X_{L} \\
\dot{X}_{L}
\end{array}\right]
\end{aligned}
$$

where,

$$
\begin{aligned}
& X_{L}=\left[\begin{array}{c}
z \\
\theta_{x} \\
\theta_{y}
\end{array}\right] \quad U_{L}=\left[\begin{array}{l}
i_{1} \\
i_{2} \\
i_{3}
\end{array}\right] \quad Y_{L}=\left[\begin{array}{l}
z_{1} \\
z_{2} \\
z_{3}
\end{array}\right] \\
& A_{L}=\left[\begin{array}{ccc}
\frac{3 K_{n}}{m_{m}} & 0 & 0 \\
0 & \frac{3 K_{n} r_{m}^{2}}{2 I_{x}} & 0 \\
0 & 0 & \frac{3 K_{n} r_{m}^{2}}{2 I_{y}}
\end{array}\right], \quad B_{L}=\left[\begin{array}{ccc}
\frac{K_{i}}{m_{m}} & \frac{K_{i}}{m_{m}} & \frac{K_{i}}{m_{m}} \\
\frac{r_{m} K_{i}}{I_{x}} & -\frac{r_{m} K_{i}}{2 I_{x}} & -\frac{r_{m} K_{i}}{2 I_{x}} \\
0 & \frac{\sqrt{3} r_{m} K_{i}}{2 I_{y}} & -\frac{\sqrt{3} r_{m} K_{i}}{2 I_{y}}
\end{array}\right], \quad C_{L}=\left[\begin{array}{ccc}
1 & -r_{s} & 0 \\
1 & \frac{r_{s}}{2} & -\frac{\sqrt{3} r_{s}}{2} \\
1 & \frac{r_{s}}{2} & \frac{\sqrt{3} r_{s}}{2}
\end{array}\right]
\end{aligned}
$$


and $i_{1} \sim i_{3}$ are currents of coils, $K_{i}$ and $K_{n}$ are force gain and stiffness of hybrid magnets at the equilibrium position, $I_{x}$ and $I_{y}$ are the moment of inertia of the MAGLEV device around the center of gravity. Since the controllability matrix of Eq.

(7) has full rank, a switching function for sliding mode control can be designed as

$$
\left[\begin{array}{l}
\sigma_{1} \\
\sigma_{2} \\
\sigma_{3}
\end{array}\right]=\left[B_{L}^{-1}\left[\begin{array}{ccc}
1 / T & 0 & 0 \\
0 & 1 / T & 0 \\
0 & 0 & 1 / T
\end{array}\right] \quad B_{L}^{-1}\right]\left[\begin{array}{l}
X_{L} \\
\dot{X}_{L}
\end{array}\right]
$$

The control law is given as

$$
U_{L}=\left[-B_{L}^{-1} A_{L} \quad-B_{L}^{-1}\left[\begin{array}{ccc}
1 / T & 0 & 0 \\
0 & 1 / T & 0 \\
0 & 0 & 1 / T
\end{array}\right]\left[\begin{array}{l}
X_{L} \\
\dot{X}_{L}
\end{array}\right]-K\left[\begin{array}{c}
\sigma_{1} /\left(\left|\sigma_{1}\right|+\delta\right) \\
\sigma_{2} /\left(\left|\sigma_{2}\right|+\delta\right) \\
\sigma_{3} /\left(\left|\sigma_{3}\right|+\delta\right)
\end{array}\right]\right.
$$

where $T$ is the time constant, $K$ is the magnitude of nonlinear input and $\delta$ is the constant for reducing chattering phenomenon.

\subsection{Levitation test}

In order to verify the effectiveness of the proposed levitation control method, levitation test was done by using developed MAGLEV device (see Fig. 3). Parameters of the designed controller are shown in Table. 3. The experimental results of levitation test with and without considering reducing chattering phenomenon are shown in Figs. 11 and 12. These experimental results can verify the effectiveness of the proposed sliding mode based levitation control method. The levitation performance, which is significant for our proposed vibration control method because of the desired normal force should be provided by controlling the levitation distance of MAGLEV device, can be guaranteed by using the designed sliding mode controller. So, integrating this levitation control method into the above mentioned vibration control system of structures, experiments were done to validate the functionality of the system further.

Table 3 Parameters in the levitation test

\begin{tabular}{c|c|c|c|c|c|c|c|c}
\hline$I_{x}\left[\mathrm{~kg} \mathrm{~m}^{2}\right]$ & $I_{y}\left[\mathrm{~kg} \mathrm{~m}^{2}\right]$ & $K_{i}[\mathrm{~N} / \mathrm{A}]$ & $K_{n}[\mathrm{~N} / \mathrm{m}]$ & $T[\mathrm{~s}]$ & $K[\mathrm{~A}]$ & $r_{m}[\mathrm{~m}]$ & $r_{s}[\mathrm{~m}]$ & $\delta$ \\
\hline $5.21 \times 10^{-4}$ & $4.70 \times 10^{-4}$ & 2.28 & $1.65 \times 10^{3}$ & 400 & 5 & 0.04 & 0.045 & 0.01 \\
\hline
\end{tabular}

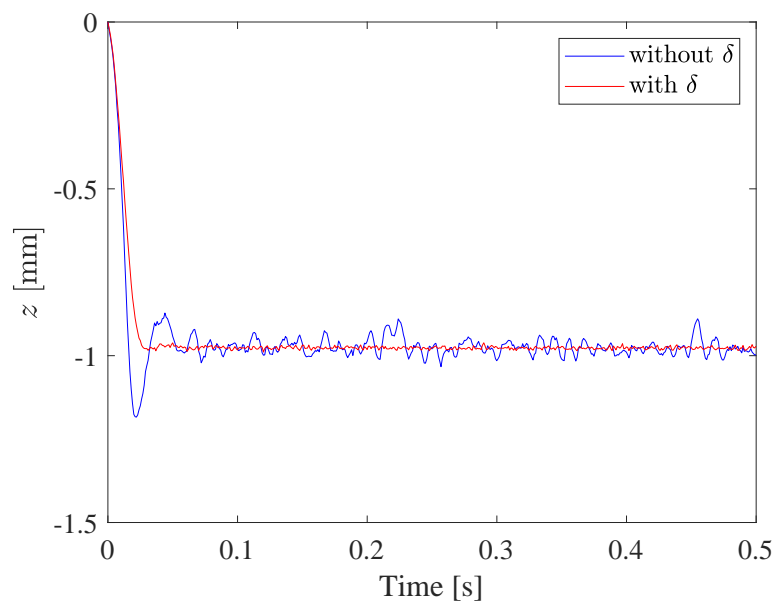

Fig. 11 Position of MAGLEV device. The position of MAGLEV device with $\delta$ is stabler than the one without $\delta$, and the overshot is also smaller than the latter.
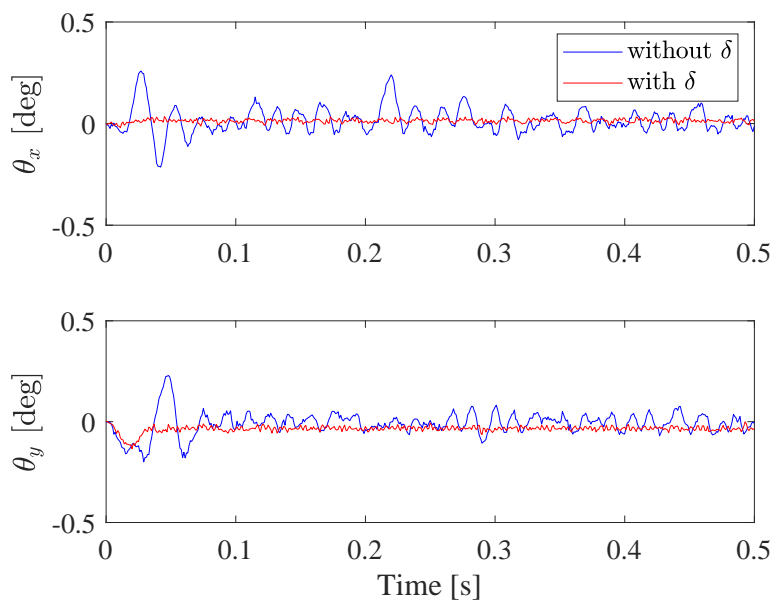

Fig. 12 Tilt angles. $\theta_{x}$ and $\theta_{y}$ are stabler by considering reducing chattering phenomenon than not.

\section{Experimental results of vibration control}

\subsection{Experiment I}

In this experiment, the effectiveness of designed vibration control system is verified. To ensure the friction mechanism of MAGLEV device be not contact to the iron plate, there should be a little gap between the tip of the friction mechanism and the iron plate when the MAGLEV device is in the levitation state. By trial and error of levitation test, we 
selected $1.4 \mathrm{~mm}$ (levitation distance of MAGLEV device) as levitation state of MAGLEV device. To make the two-story structure vibrate, we pushed the roof of the structure by hand on the $\mathrm{X}$ direction and made the maximum value of strain gauge reach around $0.0732 \mathrm{~V}$. During vibration control, we set the normal force $f_{n}=2 \mathrm{~N}$. Since the normal force can be provided by the friction mechanism of MAGLEV device, the deflection $\Delta z=1 \mathrm{~mm}$ can decided according to Eq. (2). So if the desired levitation distance is set as $0.4 \mathrm{~mm}$, the desired deflection $\Delta z$ can be realized by levitation control. The experimental results are shown in Figs. 13-15. In Fig. 13, we can see that the displacement of the roof with control converges to zero faster than the one without control. The positions of MAGLEV device are shown in Fig. 14. Considering tilt angles of MAGLEV device (see Fig. 15) together with Fig. 14, we can see that although the tilt angles were disturbed by the friction, stable levitation was achieved during vibration control. So the above results verify the effectiveness of the designed vibration control system.

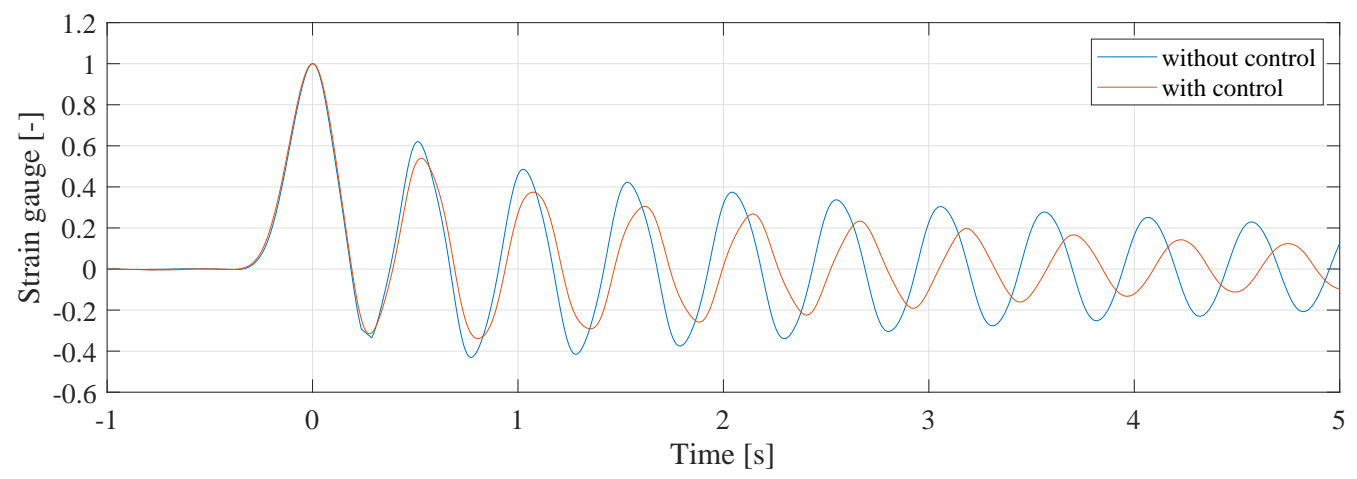

Fig. 13 Displacement of the roof. The displacement is normalized with the maximum value for a comparison with and without control.

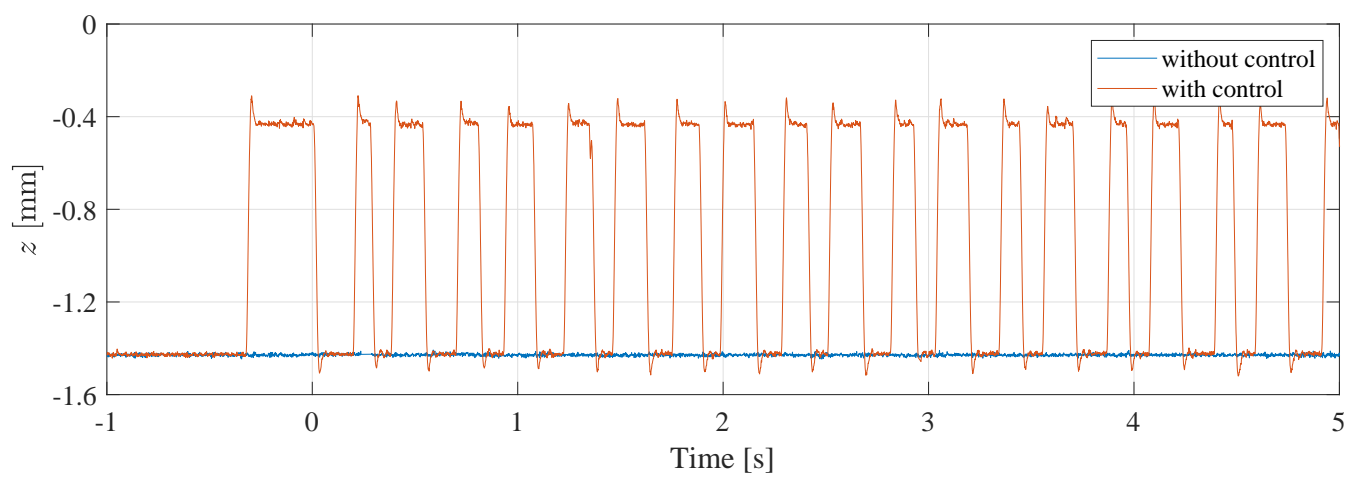

Fig. 14 Position of MAGLEV device. MAGLEV device succeeded in switching between levitation state (1.4 $\mathrm{mm})$ and contact state $(0.4 \mathrm{~mm})$ based on the proposed principle of vibration control, and provided the desired normal force to generate frictional force to consume vibration energy.

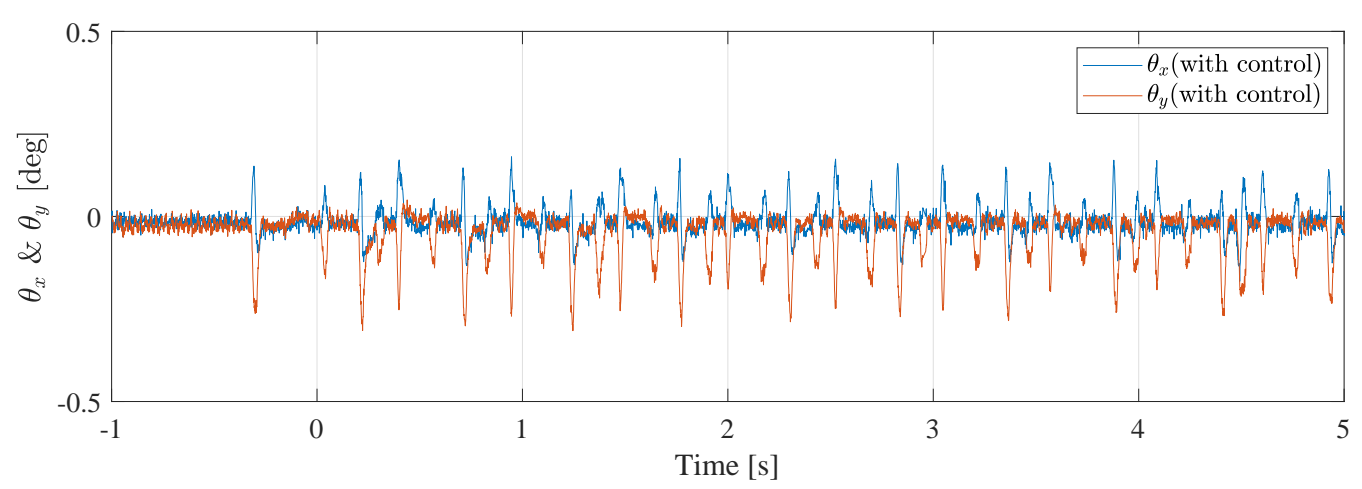

Fig. 15 Tilt angles. $\theta_{x}$ and $\theta_{y}$ could be held around 0 degree during vibration control. 


\subsection{Experiment II}

In this experiment, the effect of normal force on vibration control is investigated. We varied $f_{n}$ from $0.2 \mathrm{~N}$ to $2.6 \mathrm{~N}$ by modifying $\Delta z$ from $0.1 \mathrm{~mm}$ to $1.3 \mathrm{~mm}$ with an interval of $0.2 \mathrm{~mm}$ and obtained the position of MAGLEV device in each case (see Fig. 16). In order to compare the performance of vibration control with different normal forces, we show all the normalized displacements of the roof together in Fig. 17, and partially enlarge it so that it can be seen clearly (see Fig. 18). We also calculate damping ratio in each case by the following equation

$$
\zeta=\frac{1}{2 \pi N} \ln \frac{1}{a_{N}}
$$

where, $a_{N}$ is the amplitude of $N$ th peak. We chose $N=10$ and show the obtained damping ratios in Table 4 .

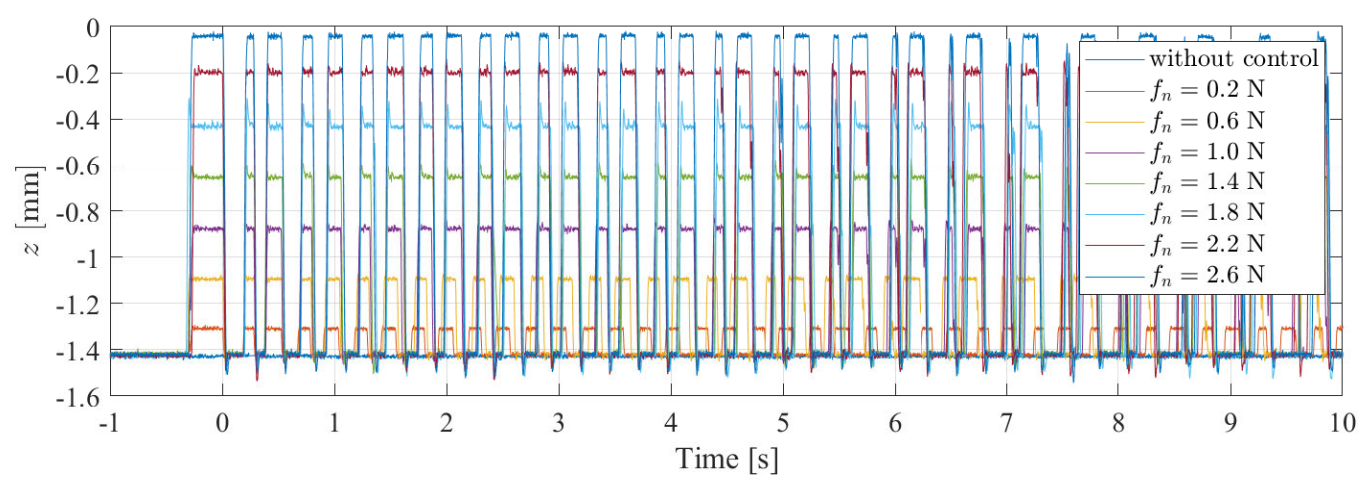

Fig. 16 Position of MAGLEV device. MAGLEV device could levitate stably at each deflection of MAGLEV device's friction mechanism to provide the desired normal force.

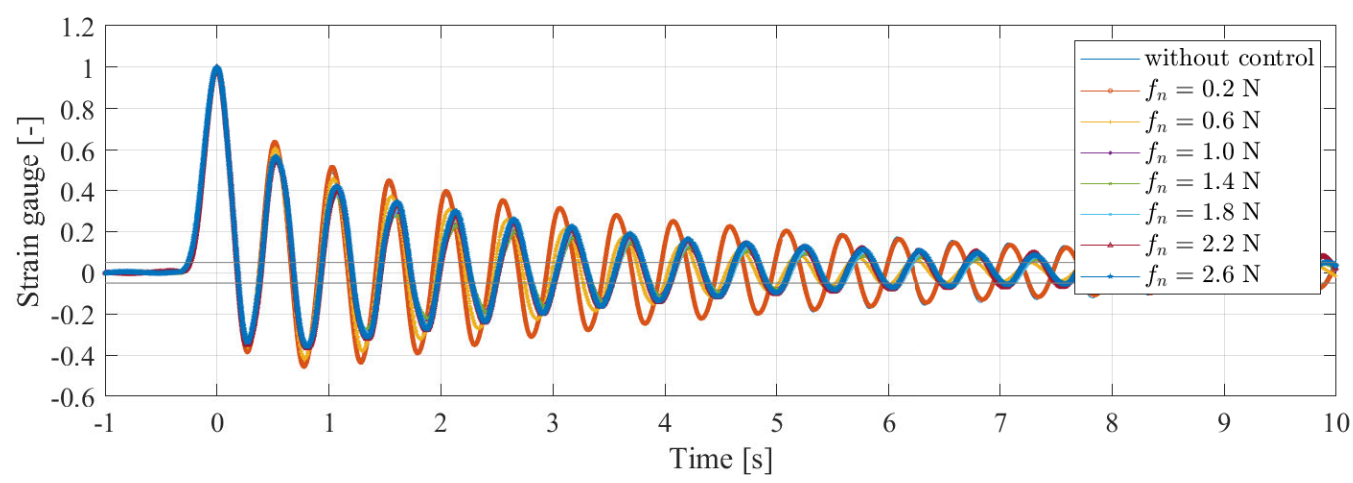

Fig. 17 Comparison on the displacement of the roof. The normalized displacement in each case is shown with different color and mark. The grey lines show the range of $\pm 5 \%$ of the maximum value.
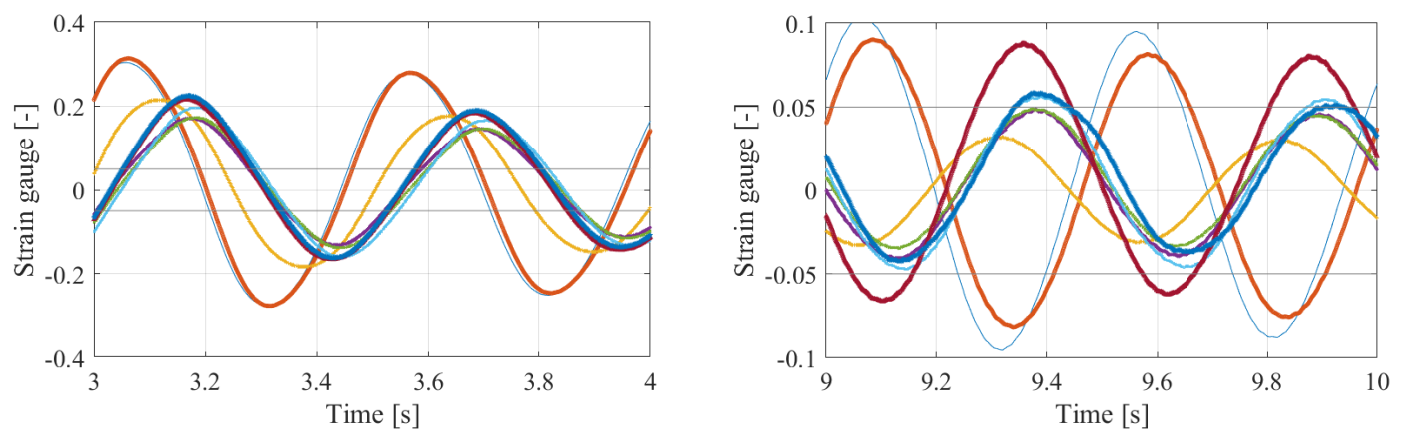

Fig. 18 Partial enlargement of Fig. 17. It is easy to see that the attenuation speed of vibration varies with different normal force.

From the above figures and table, we find that the damping ratio does not increase as the normal force increases, and the best performance of vibration control can be achieved with the normal force $f_{n}=0.6 \mathrm{~N}$. Beyond this value, the 
Table 4 Damping ratio (vibration I)

\begin{tabular}{c|c|c|c|c|c|c|c|c}
\hline$f_{n}[\mathrm{~N}]$ & 0 & 0.2 & 0.6 & 1.0 & 1.4 & 1.8 & 2.2 & 2.6 \\
\hline$\zeta$ & 0.0248 & 0.0253 & 0.0383 & 0.0376 & 0.0377 & 0.0349 & 0.0331 & 0.0327 \\
\hline
\end{tabular}

damping ratio will decrease and settling time will become longer. The reason for this phenomenon is that the consumed vibration energy depends not only on the magnitude of frictional force but also on the relative displacement between the roof and MAGLEV device. The relative displacement increased till the frictional force increases to $0.147 \mathrm{~N}\left(f_{n}=0.6 \mathrm{~N}\right)$. Beyond this value, the increase of frictional force led decrease of the relative displacement. So the above phenomenon appeared.

Further, in order to investigated whether $f_{n}=0.6 \mathrm{~N}$ is also best or not for the performance of vibration control under different strength of vibration, we pushed the roof of the two-story structure by hand on the $\mathrm{X}$ direction to exert vibration II and made the maximum value of strain gauge reach around $0.1463 \mathrm{~V}$. That is $x_{r}$ (displacement of roof) was nearly twice as big as the one of vibration I. The amplitude ratios $r_{A}$ between vibration II and I are given in Table 5. The damping ratios which are calculated with the same way as vibration I are also shown in Table 5. In order to compare the damping ratios of two vibrations, we draw them together in Fig. 19. From Fig. 19, the maximum of damping ratio shifts from $f_{n}=0.6 \mathrm{~N}$ to $f_{n}=1.0 \mathrm{~N}$. According to this result, we draw a conclusion that the optimum normal force for vibration control would vary according to the strength of the vibration.

Table 5 Damping ratio (vibration II)

\begin{tabular}{c|c|c|c|c|c|c|c|c}
\hline$f_{n}[\mathrm{~N}]$ & 0 & 0.2 & 0.6 & 1.0 & 1.4 & 1.8 & 2.2 & 2.6 \\
\hline$r_{A}$ & 2.77 & 2.02 & 2.09 & 2.07 & 1.56 & 1.65 & 2.03 & 1.95 \\
\hline$\zeta$ & 0.0276 & 0.0262 & 0.0383 & 0.0425 & 0.041 & 0.0387 & 0.0383 & 0.0361 \\
\hline
\end{tabular}

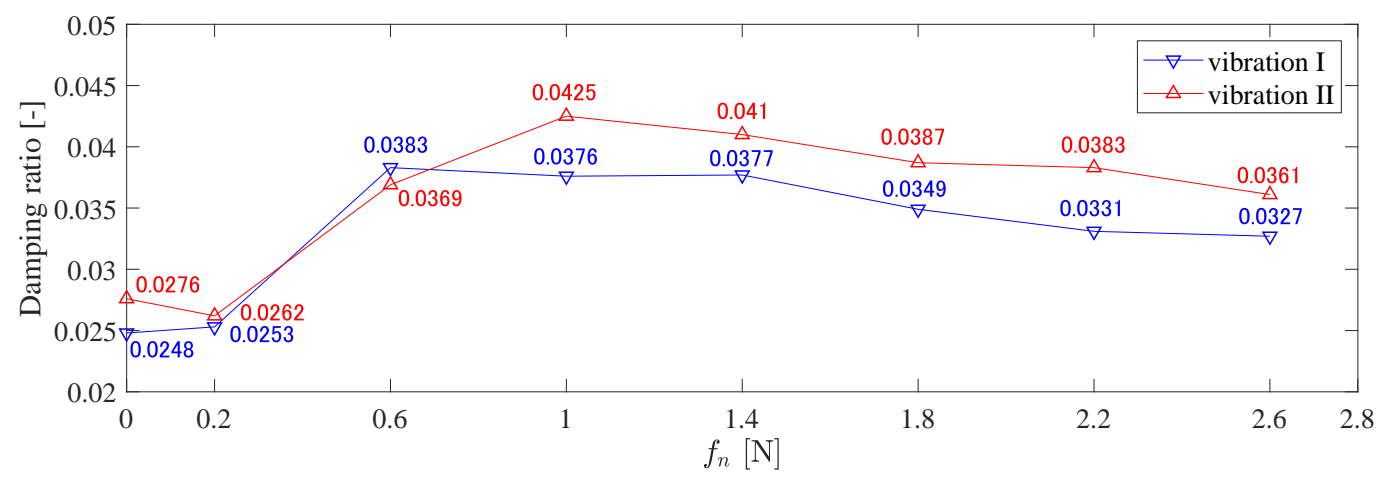

Fig. 19 Comparison of damping ratio. With the strength of the vibration increasing, the damping ratio increased. Meanwhile, the optimum normal force for vibration control shifted toward big value.

\section{Conclusion}

In this paper, vibration control system of structure was developed based on magnetic levitation technology. For generating frictional force to consume vibration energy, a MAGLEV device was developed. Based on the proposed principle of vibration control, the MAGLEV device repeated to levitate and contact to the ceiling of the structure properly. To guarantee the stability of levitation of MAGLEV device, sliding mode control method was employed. Experimental results showed the effectiveness of the designed vibration control system, and inspired us to consider optimizing the normal force according to the strength of the vibration to achieve much better performance of vibration control in the future.

\section{References}

Dihoru, L., Dietz, M., Crewe, A. J. and Tayor, C. A., Performance requirements of actuation systems for dynamic testing in the European Earthquake Engineering Laboratories, In: Fardis M., Rakicevic Z. (eds) Role of Seismic Testing 
Facilities in Performance-Based Earthquake Engineering. Geotechnical, Geological, and Earthquake Engineering, Vol.22 (2011). Springer, Dordrecht, pp.119-134.

Gutierrez, H. M. and Luijten, H., 5-DOF real-time control of active electrodynamic MAGLEV, IEEE Transactions on Industrial Electronics, Vol.65, No.9 (2018), pp.7468-7466.

Haramoto, H., Seto, K. and Koike, Y., Active vibration control of triple flexible structures arranged in parallel, JSME International Journal, Series C, Vol.43, No.3 (2000), pp.712-718.

Housner, G., Bergman, L., Caughey, T., Chassiakos, A., Claus, R., Masri, S., Skelton, R., Soong, T., Spencer, B. and Yao, J., Structural control: Past, present, and future, Journal of Engineering Mechanics, Vol.123, No.9 (1997), pp.897-971.

Jalili, N., A comparative study and analysis of semi-active vibration-control systems, Journal of Vibration and Acoustics, Vol.124, No.4 (2002), pp.593-605.

Jiang, C. and Ueno, S., Development of magnetic levitation device for active vibration control, Proceedings of the 2016 International Conference on Advanced Mechatronic Systems, Australia (2016), pp. 85-89.

Kamel, M., Kandil, A., El-Ganaini, W. A. and Eissa, M., Active vibration control of a nonlinear magnetic levitation system via nonlinear saturation controller (NSC), Nonlinear Dynamics, Vol.77, No.3 (2014), pp.605-619.

Nelson, G., Rajamani, R., Gastineau, A., Wojthiewicz, S. F. and Schultz, A. E., Bridge life extension using semiactive vibration control, IEEE/ASME Transactions on Mechatronics, Vol.20, No.1 (2015), pp.207-216.

Seto, K., Vibration control of structure, Corona Publishing Co.,Ltd (2006)(in Japanese).

Spencer, B. and Nagarajaiah, S., State of the art of structural control, Journal of Structural Engineering, Vol.129, No.7 (2003), pp.845-856.

Sun, J., Jolly, M. R. and Norris, A., Passive, adaptive and active tuned vibration absorbers-a survey, Journal of Vibration and Acoustics, Vol.117, No.B (1995), pp234-242.

Yamamoto, M., Aizawa, S., Higashino, M. and Toyama, K., Practical applications of active mass dampers with hydraulic actuator, Earthquake Engineering and structural Dynamics, Vol.30, No.11 (2001), pp.1697-1717. 